\section{ADOLESCENT MEDICINE}

1 FAMILIES OF ADOLESCENTS WITH INFLAMMATORYBOWE DISEASE:A DEMO GRAPHIC ANALYSIS. Harvey Aiges, Joseph Shore Univ. Hosp., Manhasset, N.Y., Cornell Univ. Medical Col., NY, NY Previous epidemiologic data suggest there are demographic characteristics of families which place certain individuals at higher risk for inflommotory bowel disease (IBD). To define these features in an adolescent population, parents of 79 patients with IBD ( 44 with ulcerative colitis(UC) 35 with Crohn's disease (CD)) answered questionnaires about the family constellation, and socioeconomic and medical status. These patients of whom $66 \%$ were male and $5 \%$ were non-white, were compared with 64 age and sex matched controls. No positive correlation was apparent with the parents' educational level or socioeconomic status, residential location (urban vs. suburban), or with the age rank of the patient among his or her siblings. Family history of gastrointestinal disease other than IBD was similar in the two groups, as was the incidence of intestinal carcinoma. However $20 \%$ of patients with IBD had a relative(s) with either UC or CD, compared to only $3 \%$ of controls ( $p<.05$ ). Of the 79 patients with IBD, $62 \%$ were Jewish, compared to a Jewish population of only $33 \%$ in the control group $(p<.001)$. These data suggest that Jewish adolescents, and in particular those with relatives with IBD, are ot higher risk for developing either UC or $C D$. There is no evidence that place of residence, porental education, socioeconomic status, family rank or familial occurrence of other forms of Gl disease contribute to an increased risk of IBD.

\section{2}

ESTROGEN TREATMENT OF PATIENTS WITH GONADAL DYSGENESI R. Alexander, F.A. Conte, S.L. Kaplan \& M.M. Grumbach
Dept. Pediat., Liniv. California San Francisco, Ca.

Commonly, estrogen therapy in patients with $x_{0}$ gonadal dysenesis has been deferred until 15 yrs or later; it has been inferred that treatment with estrogen at an earlier age leads to rapid skeletal maturation and a lower adult height. In order to examine this hypothesis, we studied the effect of "low dose" early conjugated estrogen therapy on peak height velocity, bone age, and helght. Seventeen patients (Croup I), 11 with a $45, \mathrm{X}$ karyotype an trogens daily starting at a 5 patients (Group II) were treated with $1.25 \mathrm{mg}$ of conjugated estrogens at a mean age of 15.5 yrs. In addition, growth was studed in 3 patients (Group III) with karyotypic evidence of a $45, \mathrm{X}$ cell line, short stature and normal gonadal function at puberty.

The mean height velocity in Group I prior to therapy was $2.7 \mathrm{~cm}$ or and post-initial estrogen therapy was $5.2 \mathrm{~cm} / \mathrm{yr}(\mathrm{p}=.0002)$; the corresponding velocities for Group II were 2.4 and $4.4 \mathrm{~cm} / \mathrm{yr}$, respectively $(p>0.5)$. The mean last measured height in Group I was $141.6 \mathrm{~cm}$ (135.4-145.8). The final height in Group II was $143.3 \mathrm{~cm}$ $(136.9-150)$, and in Group III $135.8 \mathrm{~cm}(133.5-139)$. There was no ignificant difference between these 3 groups $(p>0.1)$. These data uggest that low dose early estrogen therapy promotes secondary exual development and stimulates a growth spurt without inordinte skeletal maturation or a reduction in final height. Thus, we lyspenesis early in adolescence as it mitigates the undesirable svchologic effects of

3 GROWTH, SKELETAL MATURATION AND PUBERTY IN SICKLE CELL DISEASE. S. Castells, E.G. Kassner, N. SUNY, Downstate Medical Center, Brooklyn, New York We have previously shown that severe growth retardation in sickle cell disease is frequently associated with primary gonadal hypofunction and/or hGH deficiency. The present study was undertaken to determine the prevalence of growth retardation, retarded skeletal maturation and delayed puberty in an ambulatory clinic population: single determinations of weight age, height age, skeletal age and pubertal development (when appropriate) were made in 105 boys and girls with homozygous sickle cell disease (age range 5-17 yr). Weight age was retarded $>2$ SO in 10\% and height age was retarded $\div 2$ so in $20 \%$ (compared to standards for North American Negro children of similar socioeconomic groups); skeletal age was retarded 2 SD in $30 \%$ (compared to standards of Gruelich and Pyle). Skeletal maturation was retarded in $72 \%$ of the girls $(p \div 0.005)$ and $50 \%$ of the boys $(0.0 .01)$ with retarded height age; $56 \%(9 / 16)$ of the adolescents had delayed puberty which was associated with retarded bone age in $9 / 9$, retarded height age in $7 / 9$ and retarded weight age in 7/9. Of the $7 / 16$ adolescents with normal pubertal development, $5 / 7$ had normal height age and $6 / 7$ had normal weight age and skeletal maturation.

We conclude that a significant proportion of children with sickle cell disease have retarded growth, skeletal maturation, and pubertal development.
EFFECT OF ISOMETRIC AND DYNAMIC EXERCISE STRESS ON 4 HYPERTENSIVE ADOLESCENTS ald, Susan L. Wilson, Ruth , Pennock Laird, Victoria Fitzger Science Center, Department of Pediatrics, Dallas, Texas. Isometric (ISO) and dynamic (DYN) exercise stress tests were performed on 91 hypertensive and 42 normotensive control subjects $14-17$ years old. The hypertensives had resting systolic or diastolic pressures persistently $\geq 95$ th percentile. Blood pressure and Frank VCG were recorded during ISO handgrip (25\% of maximum effort for 4 minutes) and bicycle ergometry until the subject was exhausted. At peak DYN exercise, heart rates averaged $191 \pm 12$ (SD) bpm, and oxygen consumption $34 \pm 10 \mathrm{cc} / \mathrm{min} / \mathrm{kg}$, indicating near-maximal performance was achieved in most subjects. In the hypertensives, blood pressures (mm $\mathrm{Hg}$ ) averaged $133 \pm 11 / 77 \pm 9$ at rest, $148 \pm 13 / 92 \pm 12$ during ISO stress, and $186 \pm 21 /$ $68 \pm 21$ during peak DYN stress. In controls, pressures averaged $110 \pm 7 / 62 \pm 12$ at rest, $129 \pm 11 / 81 \pm 8$ during ISO stress, and $166 \pm 18$ / $63 \pm 14$ during peak DYN stress. During DYN stress, $34 \%$ of the hypertensives and $5 \%$ of the controls had systolic pressures $\geq 200$, however, only 1 hypertensive exceeded 220. During ISO stress, $8 \%$ of the hypertensives and none of the controls had diastolic pressures $\geq 110$; only 1 hypertensive exceeded 120 . No adolescent developed cardiovascular symptoms, arrhythmias, or ST segment displacement greater than $1 \mathrm{~mm}$. In view of these findings, we feel hypertensive adolescents should not be restricted in their physical activities unless abnormal cardiovascular changes have peen demonstrated during exercise stress testing.

SERUM 25-HYDROXYVITAMIN D (250HD) LEVELS IN CHILDREN 5 AND ADOLESCENTS WITH INFLAMMATORY BOWEL DISEASE (IBD) Rosen, (Spon. by M.I. Cohen), Albert
flore Hosp. and Med. Ctr. and Cornell Univ. Med. Coll., North flore Hosp. and Med. Ctr. and Cornell Un
Shore Univ. Hosp., Dept. Ped., New York.

Patients with IBD have abnormalities in bone growth and mineralization. Malabsorption and drug treatment may affect ca1clum and vitamin D homeostasis. 53 patients with IBD were evs1uated for abnormalities in mineral metabolism. Diagnosis, extent of involvement, and activity of disease were determined by clinical, xray, endoscopic, and blopsy data. 17 had ulcerative colitis (UC); 36 had regional enteritis (RE) with disease confined to the colon in only four. All patients had normal serum levels of total calclum, inorganic phosphorus, alkaline phosphatase, and transaminases. Serum 25OHD level in $\mathrm{ng} / \mathrm{ml}$ (mean \pm 1SEM) for all patients with UC was $24.8 \pm 1.2$ (normal 15-35). The levels of $250 \mathrm{HD}$ in UC patients with more severe disease and/ or sterold treatment did not differ from those in remission. In contrast, patients with RE of moderate activity had a mean serum 25OHD level $(14.4 \pm 2.6)$ which was significantly different from RE patients in remission $(25.0 \pm 1.7, p<0.01)$ or with mild disease $(23.9 \pm 3.0, p<0.01)$. RE patients being treated with sterolds had a mean level of $250 \mathrm{HD}(18.6 \pm 3.1)$ significantly lower than patients being treated with azulfadine alone $(25.3 \pm$ $2.1, \mathrm{p}<0.05)$.

These data Indicate that IBD of the colon does not affect vitamin $D$ homeostasis; whereas significant small bowel involvement is assoctated with low serum $250 \mathrm{kn}$ levels 